\title{
Seismic energy dispersion compensation by multi-scale morphology
}

\author{
Yu Junqing ${ }^{1}$, Wang Runqiu ${ }^{1 *}$, Liu Taoran ${ }^{2}$, Zhang Zhenglong', Wu Jian', \\ Jiang Yongyong ${ }^{1}$, Sun Lipeng ${ }^{1}$ and $\mathrm{Xia} \mathrm{Pei}^{1}$ \\ ${ }^{1}$ College of Geophysics and Information Engineering, China University of Petroleum (Beijing), Beijing 102249, China \\ ${ }^{2}$ BGP Research \& Development Center, Hebei 072751, China
}

(C) China University of Petroleum (Beijing) and Springer-Verlag Berlin Heidelberg 2014

\begin{abstract}
Seismic energy decays while propagating subsurface, which may reduce the resolution of seismic data. This paper studies the method of seismic energy dispersion compensation which provides the basic principles for multi-scale morphology and the spectrum simulation method. These methods are applied in seismic energy compensation. First of all, the seismic data is decomposed into multiple scales and the effective frequency bandwidth is selectively broadened for some scales by using a spectrum simulation method. In this process, according to the amplitude spectrum of each scale, the best simulation range is selected to simulate the middle and low frequency components to ensure the authenticity of the simulation curve which is calculated by the median method, and the high frequency component is broadened. Finally, these scales are reconstructed with reasonable coefficients, and the compensated seismic data can be obtained. Examples are shown to illustrate the feasibility of the energy compensation method.
\end{abstract}

Key words: Seismic wave, multi-scale morphology, dispersion compensation, high resolution, median method, spectrum simulation

\section{Introduction}

With the rapid development of industrial economies, exploration for oil and gas fields has come to a new stage. The improvement of seismic data resolution has become an important issue in geophysics and it has also attracted much attention in China. The energy of seismic waves decays as seismic waves propagate, which results in low resolution of seismic data (Wang and Sun, 2012). Necessary means should be taken to obtain seismic sections with high resolution, not only by high precision broad-band data field acquisition, but also by appropriate later processing. During the processing of seismic data, reasonable compensation of high frequency component lost in seismic data is most important to improve its resolution (Li and Wang, 2007; Yuan and Wang, 2013). So research on this topic has significant theoretical and practical value.

The most common method for compensation is an inverse- $Q$ filter (Yu, 1993) which is the reverse of forward wave propagation. It attempts to compensate for absorption of seismic waves, and is important for improving resolution (Wang, 2008). However, it requires an accurate $Q$ value which is often difficult to calculate. So the compensation effect is restricted by the experience and skill of the user.

*Corresponding author. email: wrq@cup.edu.cn

Received April 6, 2013
As well, the inverse- $Q$ filter is time-varying which can bring some difficulties to filtering (McCarley and Lon, 1985). Furthermore, different layers have different levels of absorption, so it is difficult to compensate them well. Another common compensation method is spectral whitening ( $\mathrm{Li}$, 1993). In this method, time windows should be opened at regular intervals, and then spectral whitening processing is operated in each window. However, the same compensation coefficient within the time windows is a disadvantage. Larger time windows will bring insufficient compensation to deep layers, while smaller time windows will make the reflection coefficient statistical properties disappear. When the reflection coefficient is not white noise, spectral whitening processing will damage the reflection coefficients (Liu and Wang, 2013). In addition, spectral whitening frequency decomposition and reconstruction is a non-orthogonal transformation, so it is difficult to describe the relative relationship between amplitudes of the spectral whitening processed seismic data, which is unfavorable for subsequent processing and high precision interpretation.

Moreover, a simple compensation method in the frequency domain, an energy attenuation compensation method based on wavelet packet decomposition ( $\mathrm{Li}$ et al, 2000), a new method for inverse- $Q$ filter in the depth domain, a spherical divergence and compensation method of absorption in the time-frequency spatial domain and a compensation method of attenuation by formation absorption based on a generalized 
S transform (Liu et al, 2006) are proposed for compensation. Researchers have also proposed other methods, such as wave equation extension along the ray path (Ren et al, 2007), a stable inverse $Q$ migration method (Zhang and Wang, 2010), a compensation method based on an improved generalized S transform (Zhang et al, 2010), a method of compensation for propagation losses based on a one-way propagator in the mixed domain (Sun and Fu, 2012), high angle pre-stacking migration compensation method (Zhou et al, 2012) and an inverse $Q$ filter method based on the Malvar spectrum ( $\mathrm{Zi}$ and Gai, 2012).

The method in this paper is different from the previous ones. Seismic waves have different waveforms, and mathematical morphology method is capable of seeking the tiny differences between them, so different sections categorized by their waveforms can be obtained, which is called multiple scales decomposition. In this paper, seismic data is decomposed into multiple scales while maintaining the previous geological characteristics, and then the high frequency components are compensated while the middle and low frequency components are also adjusted in some scales by the method of spectrum simulation. The continuation frequency band is determined with median method. Eventually, the coefficients of reconstruction in each scale should be determined and the seismic record, in which seismic energy is compensated, the effective frequency band is broadened and resolution is improved, can be obtained by data recombination. Combination of multi-scale morphology and the spectral simulation method for seismic energy dispersion compensation is the core content of this paper.

\section{Seismic energy dispersion compensation by multi-scale morphology}

Attenuation compensation, which corrects the attenuation and dispersion of seismic waves, is one of the effective methods for improving seismic data resolution (Wang, 2011). Compensation of seismic energy determines the resolution of seismic data. Like high resolution processing, compensation of seismic energy is always important in geophysics. Rational energy compensation of seismic data has become one of the main directions of seismic data processing. Conventional seismic compensation methods of improving the resolution of seismic data at the same time worsen the signal-to-noise ratio (SNR). To remove this limitation, multi-scale morphology and spectral broadening are combined to improve the resolution, and meanwhile minimize the influence on SNR. In this section, application of multi-scale morphology and spectral broadening in seismic data processing will be discussed. In the multi-scale sections of morphology, seismic data resolution is improved by Fourier analysis and seismic energy is compensated by broadening the frequency spectrum.

\subsection{Multi-scale morphology in seismic data processing}

Structure elements of mathematical morphology play a vital role in the shape feature extraction of the signal and image (Chen et al, 2009), but a structure element with a given shape can only handle target bodies which have big differences in shape but cannot handle target bodies which have similar shapes and different sizes. So another property, scale, should be introduced. Given a structure element sequence in which all structural elements have the same shape but different sizes we then can process the signal and image according to the shape and size of structural elements at the same time. This algorithm which uses different scales of structural elements for morphological transformation is called multi-scale morphology (Li, 2005).

As mathematical morphology has useful properties, it was introduced into seismic data digital processing (Zheng and Wang, 2003). With the intensive research on mathematical morphology, multi-scale morphology has a wide range of applications in seismic data processing. It can be applied to suppressing surface waves and interfering waves (Wang et al, 2008), detecting seismic fractures (Chen et al, 2008), and removing multiple waves (Wang, 2008a). Furthermore, Luo et al (2011) processed LiDAR data with a filter method based on morphology. Zhang et al (2012) achieved seismic data amplitude compensation by multi-scale morphology.

\subsection{Principle of compensation of seismic energy dispersion with multi-scale morphology}

Multi-scale decomposition can be done according to the characteristics of seismic waveform differences (Goutsias and Heijmnas, 2000) which can be found by mathematical morphology even when the difference is small. In this paper, the seismic section will be decomposed into different scales based on a multi-scale mathematical morphology decomposition algorithm (Mukhopadhyay and Chanda, 2002). Since the desired wave and the interference wave exist at different scales, desired signal and noise can be better distinguished in each scale section. So, the effective band can be broadened with a spectrum simulation method in some selective scales. Then the multi-scale morphological reconstruction algorithm principle can be used to analyze the SNR in each scale section, which can be reconstructed by various coefficients in each scale section, for instance, the high frequency and low frequency components should be suppressed by small coefficients, and the final seismic section after energy compensation can be eventually obtained (Koskinen et al, 1991).

We should pay attention to the following points during the compensation of seismic energy dispersion.

\section{1) Basic principle of compensation for energy dispersion} by the spectral expansion method

The main method to broaden the spectrum is selecting the seismic trace with a small offset and high SNR as standard trace by noise analyses of each trace in the seismic records, transforming the standard trace from the time domain to the frequency domain and using the spectrum simulation method to fit middle and low frequency components and compensate high frequency components. Then apply the high frequency compensated curve to traces and the seismic section after energy compensation has been achieved. This compensation method has an advantage that only the high frequency component should be compensated while the main frequency 
information is retained, so the effective frequency band can be broadened and the resolution of the signal is improved.

The three assumptions of the spectrum simulation method are that amplitude spectra of the actual seismic wavelet are similar in time and space; the amplitude spectra of the seismic record and wavelet are similar and the amplitude spectrum of the seismic wavelet is continuous and smooth.

Fitting a polynomial in spectrum simulation is:

$$
y(f)=f^{2} \mathrm{e}^{\sum_{n=0}^{L} a_{n} f^{n}}
$$

where $y$ is amplitude energy; $f$ is frequency and $a_{n}$ is the point value which means the median approximation value.

The variance norm criterion, Wiggins variance norm formula, which is used to select the fitting frequency range (Wiggins, 1985; Yuan and Wang, 2011) is as follows:

$$
V_{\operatorname{arimax}}=\frac{\sum_{i}^{N} x_{i}^{4}}{\left[\sum_{i}^{N} x_{i}^{2}\right]^{2}}
$$

where $x_{i}$ is the $i$ th value in the seismic trace data. Given the initial frequency $\mathrm{f} 1$, then select frequency $\mathrm{f} 2$ corresponding to the maximum $V_{\text {arimax }}$, therefore, we can get the frequency band (f1-f2) which is the best simulating band. The compensation effect of expansion processing that fits with this band is optimum.

\section{2) Choice of fitting frequency band}

During the expanding compensation, the simulation band (f1-f2) should be determined by the spectral features of the seismic data. After several experiments, it can be found that when the range is (f1-f2), f1 should be about $5-10 \mathrm{~Hz}$, so that authenticity of fitting middle and low frequency components can be kept; f 2 should be chosen about 5-10 Hz higher than the main frequency to get an appropriate compensation effect. Meanwhile, the range should be adjusted according to the actual spectrum figure.

\section{3) Choice of spectral expansion band}

Different regions have different seismic data quality, so the expansion range has a restriction. According to

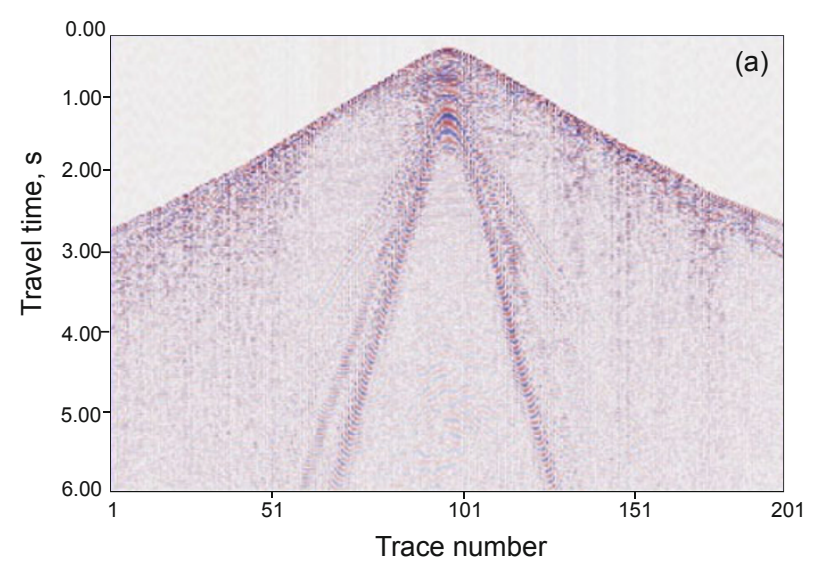

the analysis of the ranges of the minimum and maximum effective frequency in spectrum, the cut-off frequency of the high frequency expansion, f3, can be determined. When out of the range, the SNR of the seismic data will drop seriously. 4) Determination of fitting curves by the median method

In field processing, the spectrum after Fourier transform is not smooth, but fluctuates up and down. For this reason, fitting curves have big differences in different intervals which cause a lot of trouble for follow-up work. To solve this issue, the median method can be used to select the best smooth fitting curve to make sure that it cannot vary with different choices of starting point.

This example shows an original seismic record that has 201 traces, 3,501 sampling points and $2 \mathrm{~ms}$ sampling interval. It can be seen from Fig. 1 that the spectrum is not smooth but fluctuates up and down which means that fitting curves have big differences with different starting points. The following figures (Fig. 2) show the comparison of five fitting curves in and around $5-90 \mathrm{~Hz}$, and the median fitting curve Fig. 2(f) is obtained by processing these five curves with median method.

It can be seen from Fig. 3 that fitting curves by the median method cannot be influenced by the choice of interval. Therefore, we can get a stable smooth fitting curve by the median method and avoid the influence of the differences of fitting curves in different intervals. So the fitting curve should be determined by the median method.

\subsection{Flow chart of compensation of seismic energy dispersion by multi-scale morphology}

During the energy compensation, spectral expansion will reduce the SNR. Multi-scale morphology is introduced to reduce the influence from processing in each scale. Combination of multi-scale morphology and the spectral expansion method to compensate seismic energy can reduce the influence on the SNR while improving resolution. Based on the analysis above, high frequency compensation of seismic data can be achieved by the following steps (Fig. 4).

1-96 Hz, 3-98 Hz are selected for direct curve fitting and compared with the median fitting curve.

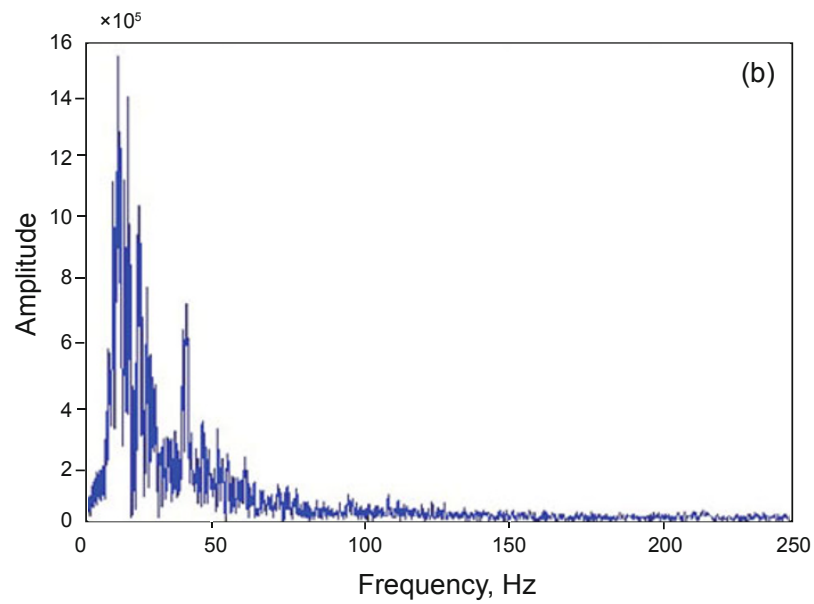

Fig. 1 Original record and spectrum

(a) Original shot record (b) Spectrum after Fourier transform of one trace 

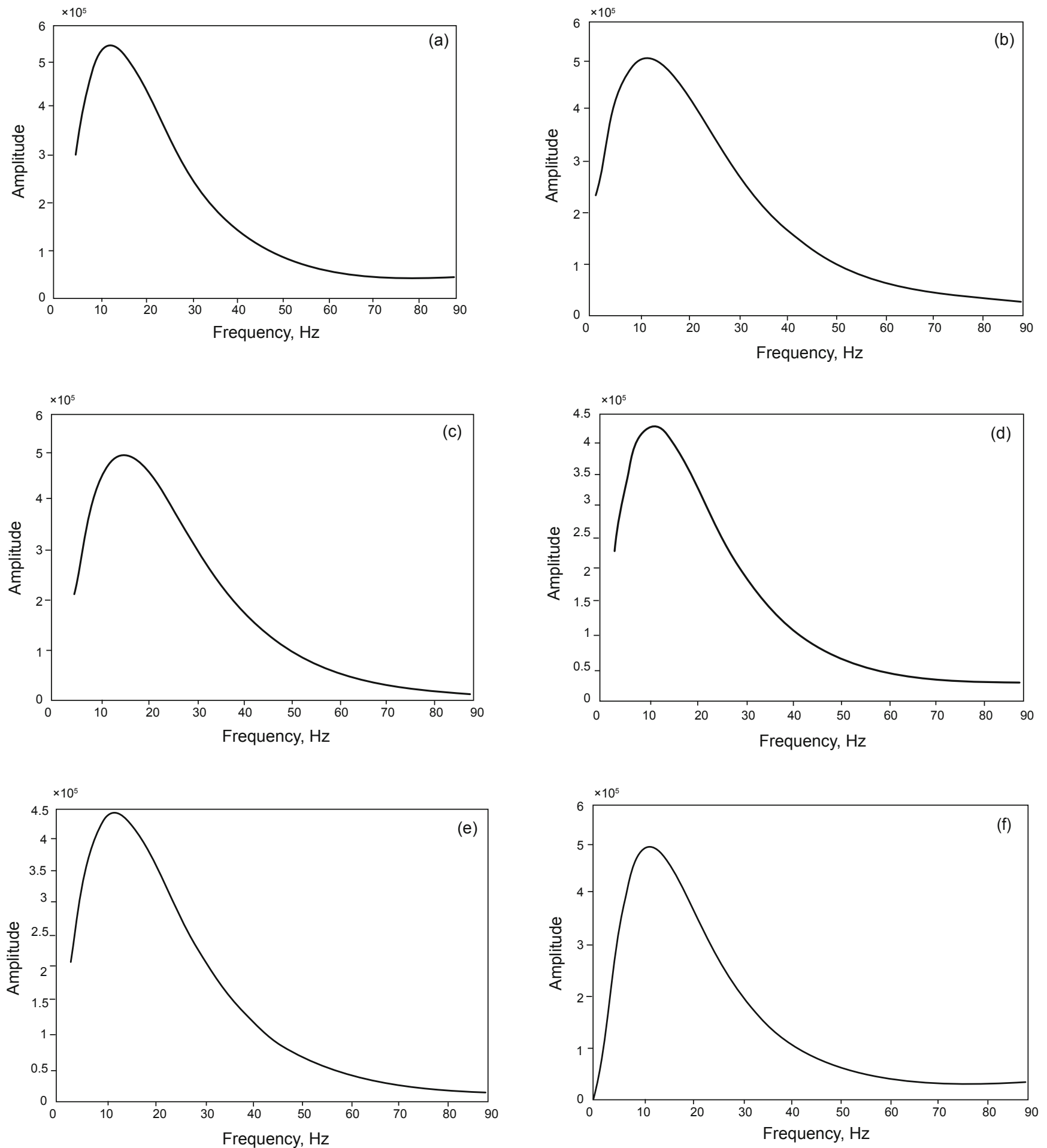

Fig. 2 Fitting curves

(a) Fitting curve in $5-90 \mathrm{~Hz}$ (b) Fitting curve in 6-86 Hz (c) Fitting curve in 7-87 Hz (d) Fitting curve in $8-88 \mathrm{~Hz}$ (e) Fitting curve in $9-89 \mathrm{~Hz}$ (f) Median fitting curve

\section{Analysis of examples}

An experiment with the Geoeast processing and interpretation system on an original shot record with this method is as follows (Fig. 5 and Fig. 6).

At first, we decompose the original shot record into five scales using the multi-scale morphology method.

It can be seen from Fig. 7 that sections at small scale show the details of the original seismic section while sections of large scale show the whole trend of original seismic section. With different information in different scales, information contained in the seismic signal can be utilized more fully. The multi-scale sections can be also applied in seismic interpretation to identify the geological phenomenon which is difficult to observe in the seismic sections, such as the location of pinch out, low amplitude structures and small 


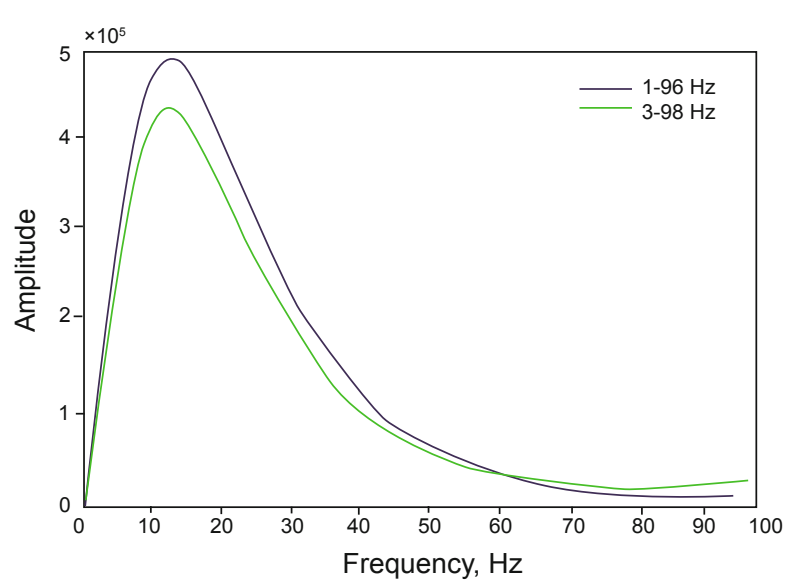

Fig. 3 (a) Direct fitting curve in $1-96 \mathrm{~Hz}$ and $3-98 \mathrm{~Hz}$

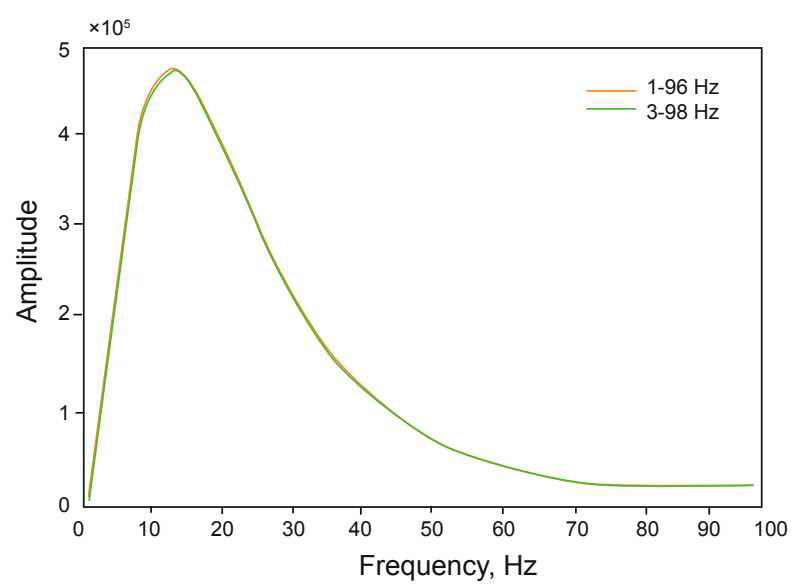

Fig. 3 (b) Median fitting curve in $1-96 \mathrm{~Hz}$ and $3-98 \mathrm{~Hz}$

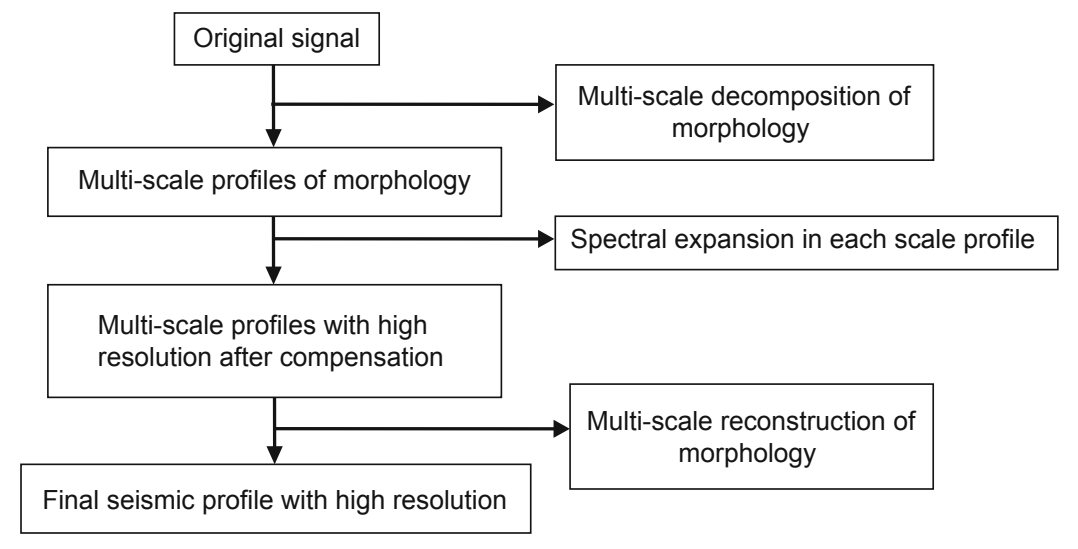

Fig. 4 The flow chart of energy compensation by multi-scale morphology and spectral expansion

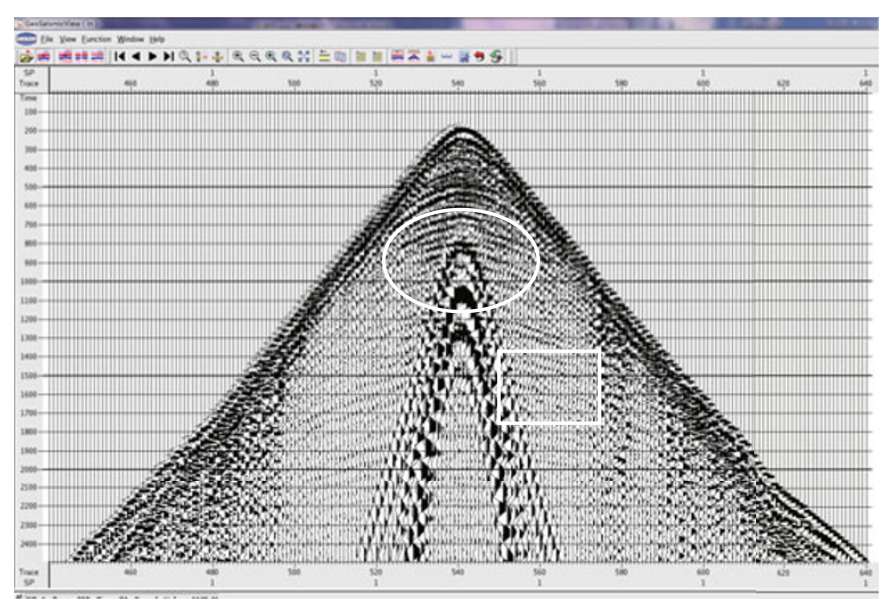

Fig. 5 Original shot record

faults.

Fig. 8(a-e) are spectrums from scale 1 to scale 5 .

Spectral analysis of multi-scale sections shows that the width of the spectrum becomes smaller from small scale to large scale, and the scale 1 section has the widest spectrum and contains almost the whole frequency band range. It is reasonable that the width of spectrum becomes smaller as the

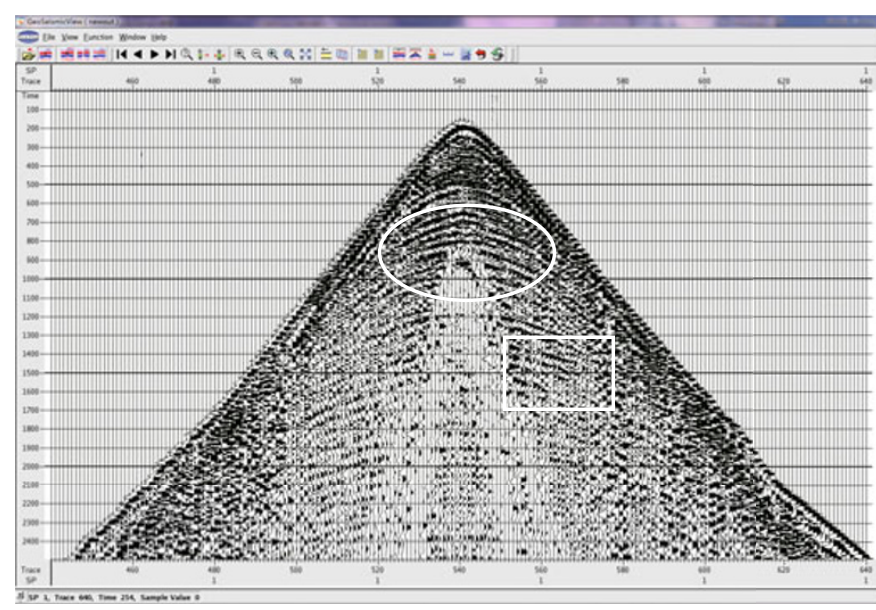

Fig. 6 Record after multi-scale morphology processing

scale increases. It means that effective bandwidth of section is determined by the scale of the structural operator.

As shown above, it can be deduced that the spectrum component is integrated in the section of scale 1 , so there is no need to process it because over compensation will cause high frequency noise. There are almost all low frequency components which are mainly surface wave signals in scale 

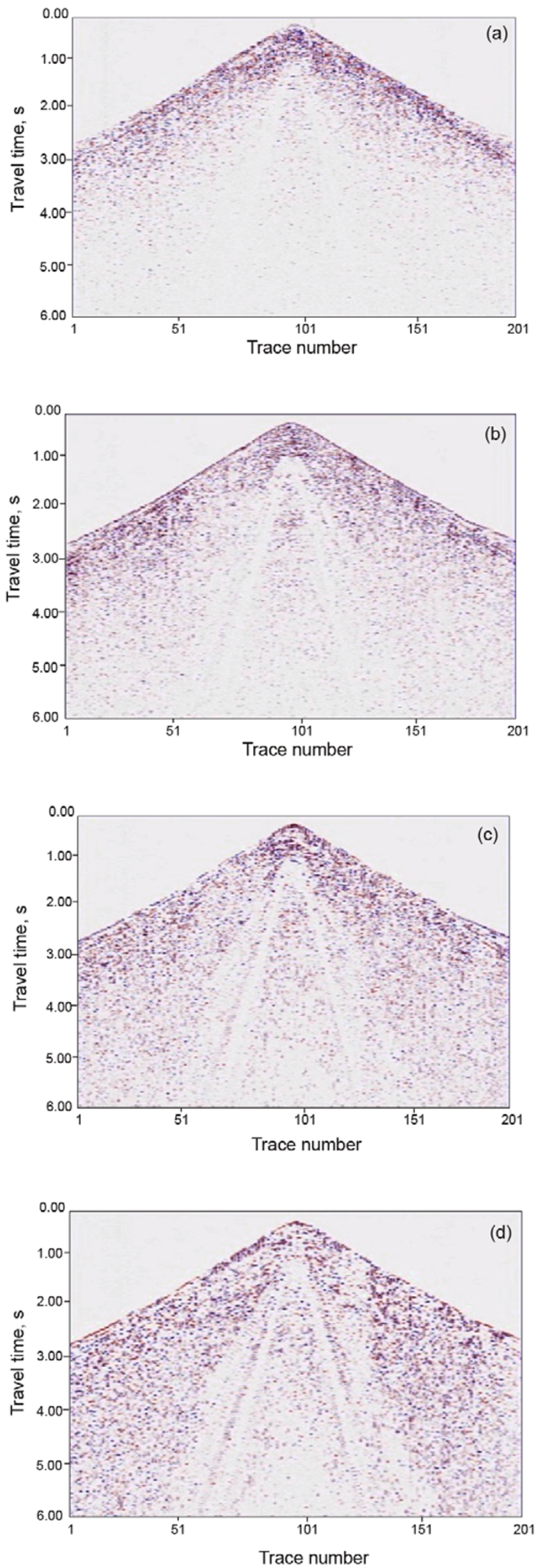

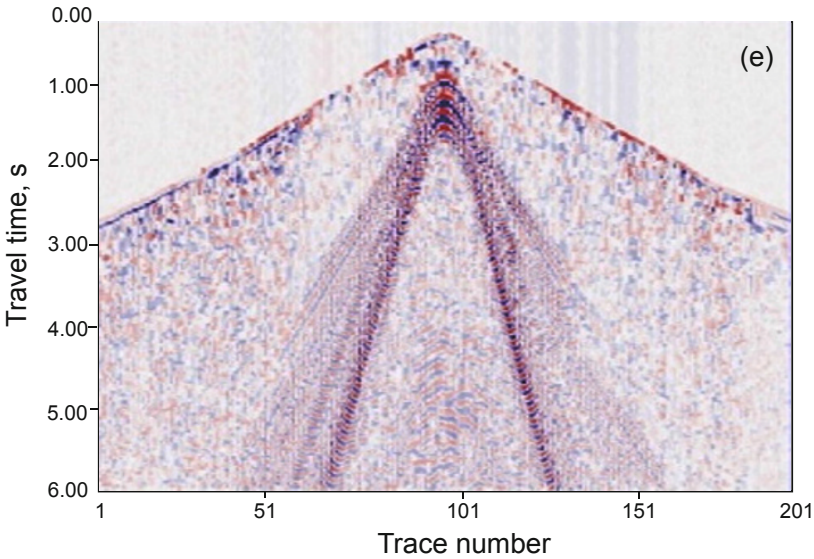

Fig. 7 Five seismic sections after multi-scale morphology processing (a) Section of scale 1; (b) Section of scale 2; (c) Section of scale 3; (d) Section of scale 4; (e) Section of scale 5

5 , so there is no need to process it either. The following figure shows the process of effective frequency expansion of scale 2, 3 and 4 by spectrum simulation (Fig. 9). Simulation curves can be received by low frequency signals, based on which the
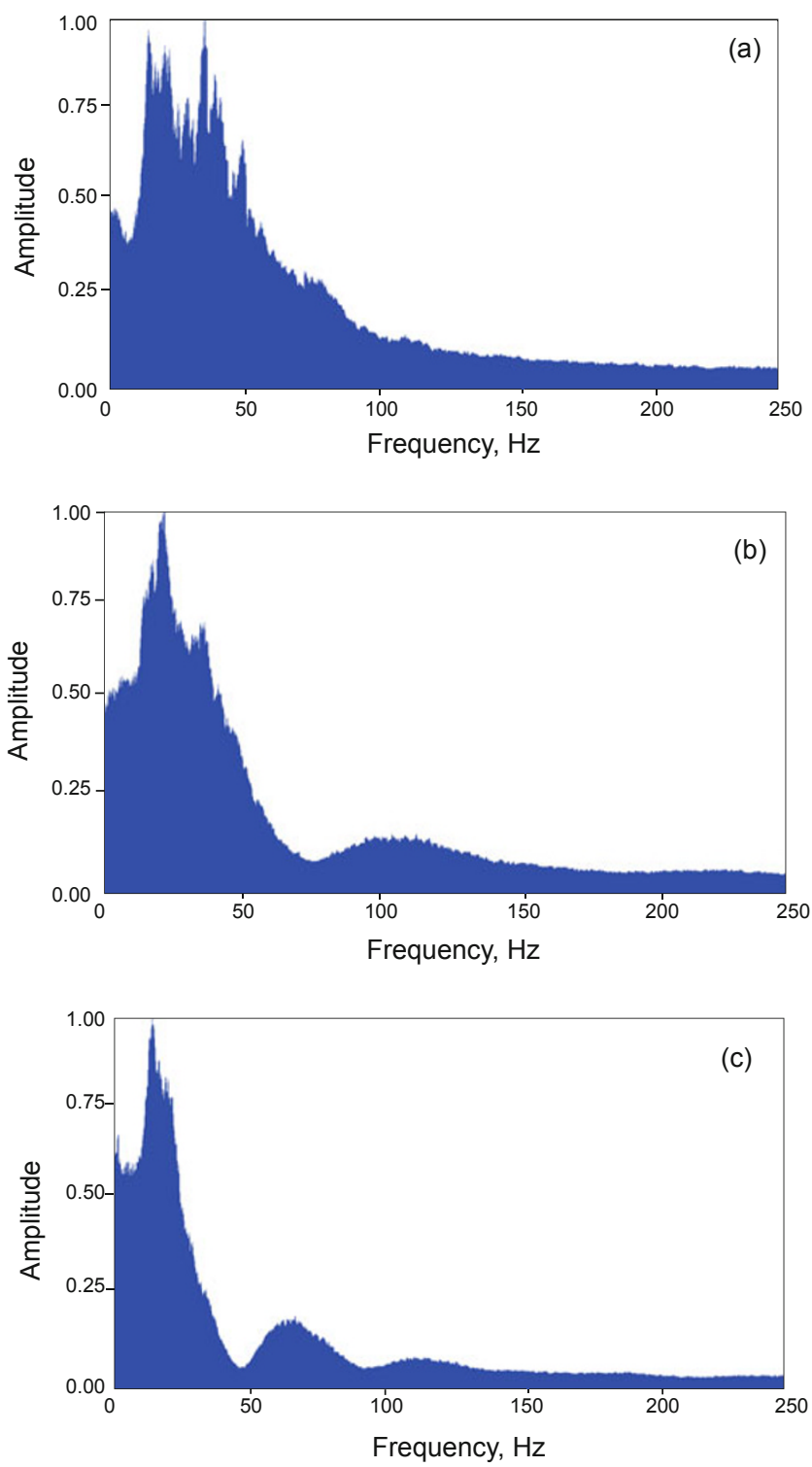

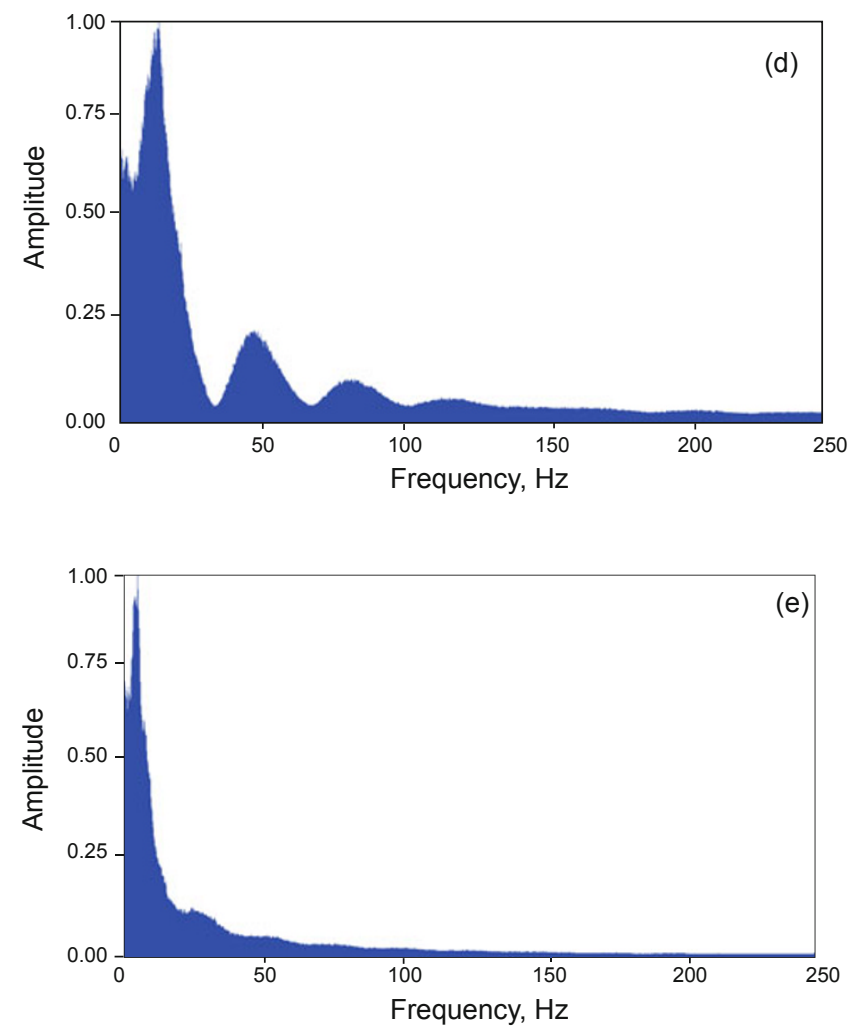

Fig. 8 Amplitude spectra of each scale

(a) Amplitude spectra of scale 1; (b) Amplitude spectra of scale 2; (c) Amplitude spectra of scale 3; (d) Amplitude spectra of scale 4; (e) Amplitude spectra of scale 5

high frequency part can be extrapolated and then be weighted stacked with the original high frequency signal.

Finally, each scale can be reconstructed. Coefficients of scale 1 to 4 are 1 while 0.05 in scale 5 because it contains mostly surface signal that has to be suppressed.

Figs. 10 and 11 show a comparison of the amplitude spectrum of the seismic section before and after multi-scale morphology and spectral expansion processing.

Comparing Fig. 5 with Fig. 6, we can see that after multiscale morphology processing, the effect of suppressing surface waves is good. From the comparison of the oval and rectangular areas between the two figures, it can be found that after the spectral expansion processing, the effective frequency band is broadened, the high frequency component is compensated, the events become clearer and their continuity has been improved. Comparing Fig. 10 with Fig. 11, it can be also found that the effective frequency band of the section is broadened by the spectrum simulation method. Above all, after morphology and spectral expansion processing, the seismic energy is strengthened, the frequency band becomes wider and the event becomes thinner.

The original shot record (Fig. 12) should be decomposed with multi-scale morphology into nine scales, and then using appropriate reconstruction coefficients, the section after multi-scale morphology processing is shown in Fig. 13. Comparing Fig. 12 with Fig. 13, it can be found that after multi-scale morphology processing, the SNR has been improved, especially in the surface wave suppression. In the
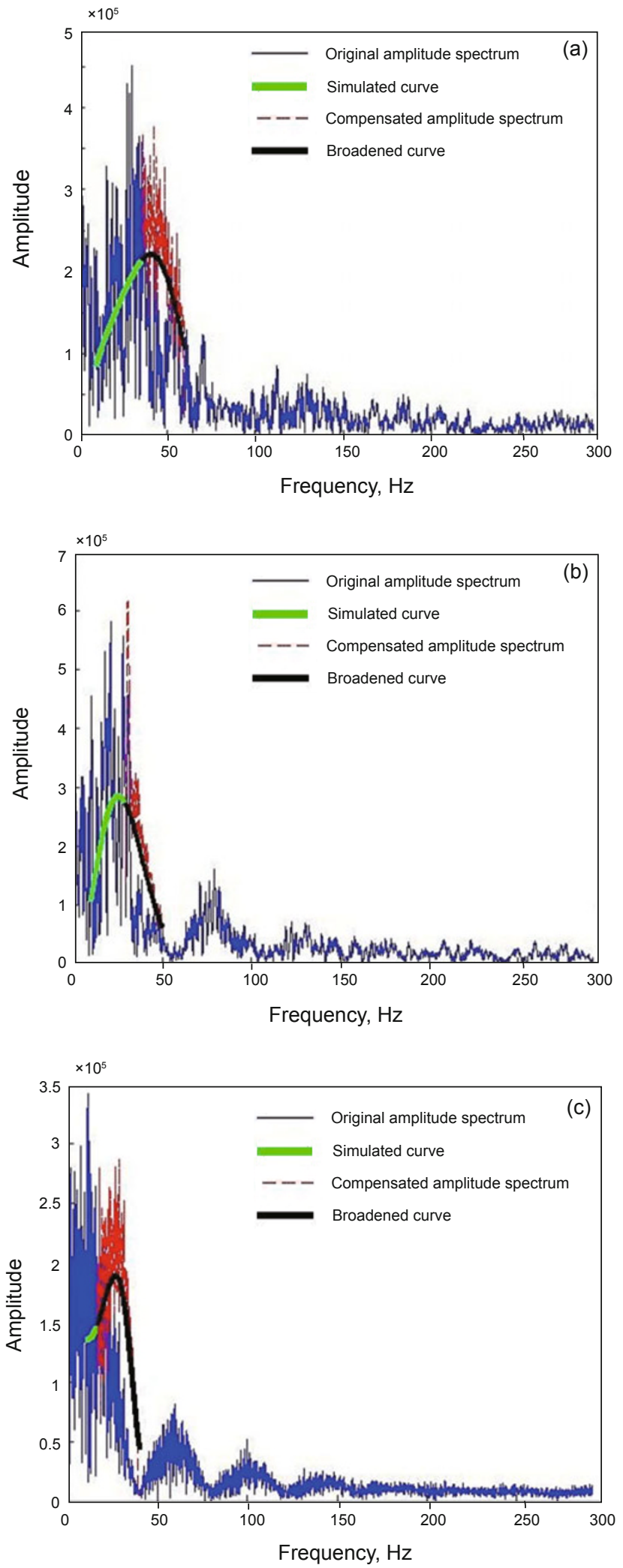

Fig. 9 Broadening of frequency spectra (a) Frequency spectra of scale 2 (b) Frequency spectra of scale 3 (c) Frequency spectra of scale 4

regular de-noising method, the resolution will decrease with improvement of the SNR. However, this problem can be solved in mathematical morphology processing, because the seismic data is processed in full frequency band. 


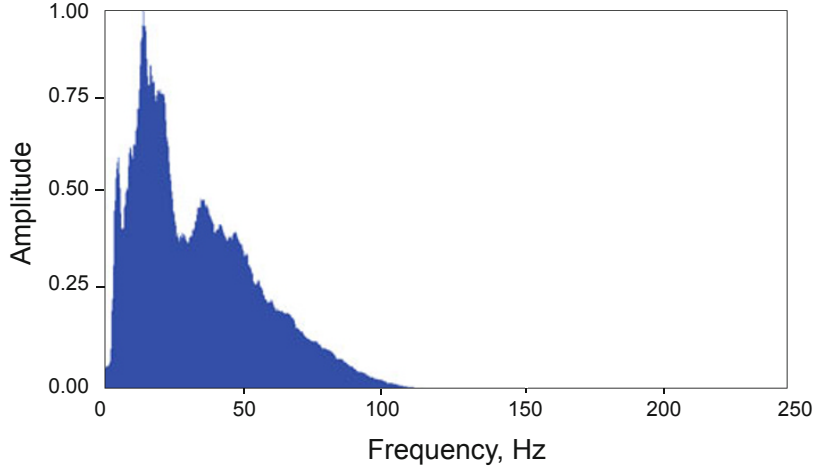

Fig. 10 Amplitude spectrum of original section

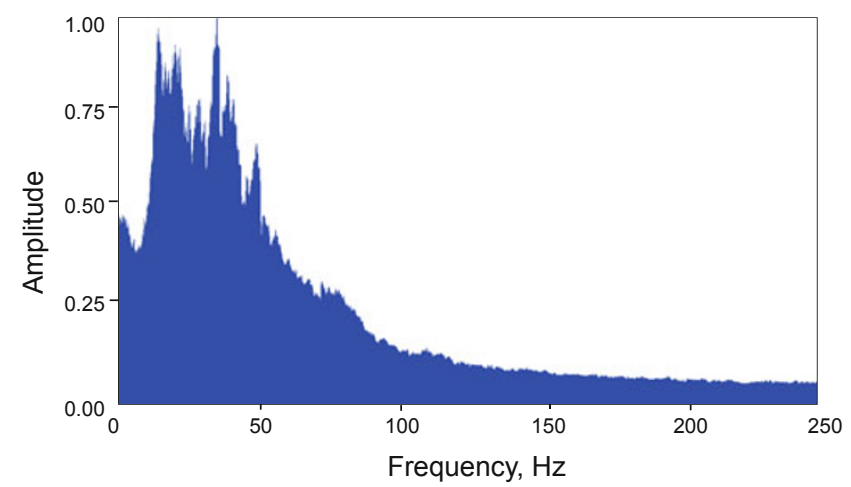

Fig. 11 Amplitude spectrum of section after morphology processing

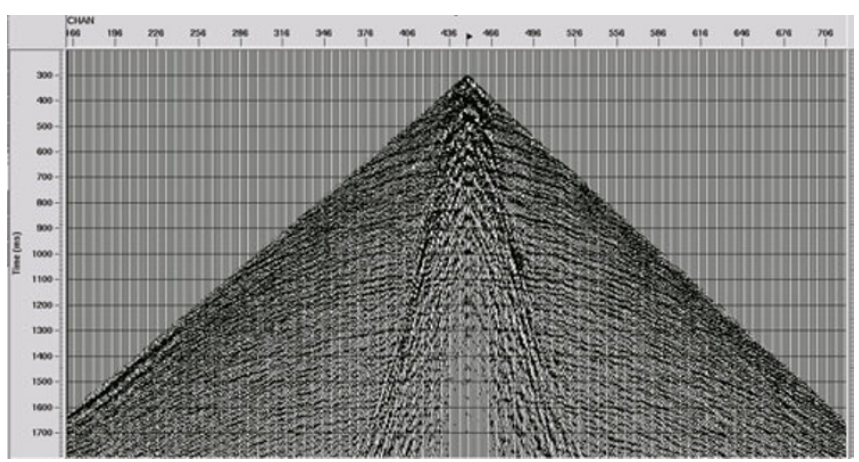

Fig. 12 Original shot record

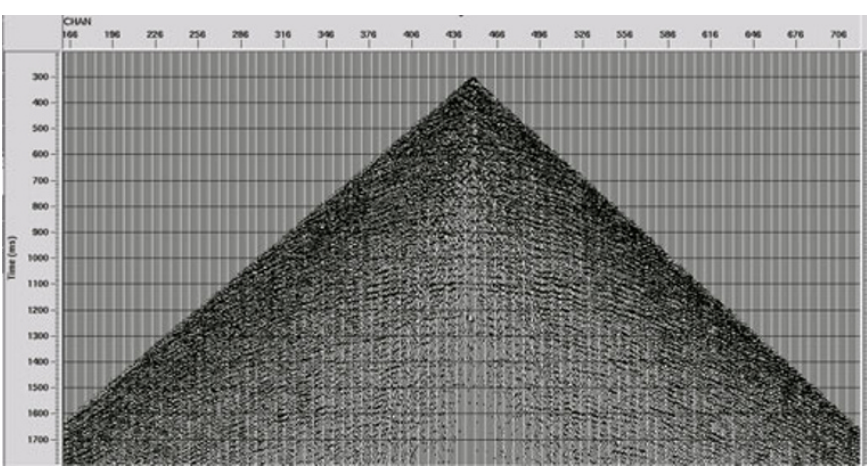

Fig. 13 Record after multi-scale morphology processing

Compared with Fig. 13 in the bigger oval area of Fig. 14(a), the resolution of the section has been improved. Compared with Fig. 14(a), in the Fig. 14(b), after the combination processing, the damage to the signals caused by the suppression of surface waves has been made up, because marks left by the suppression of surface waves has been removed by the spectrum continuation compensation. Furthermore, the continuity of seismic events, as well as resolution, have been improved in the smaller oval area.
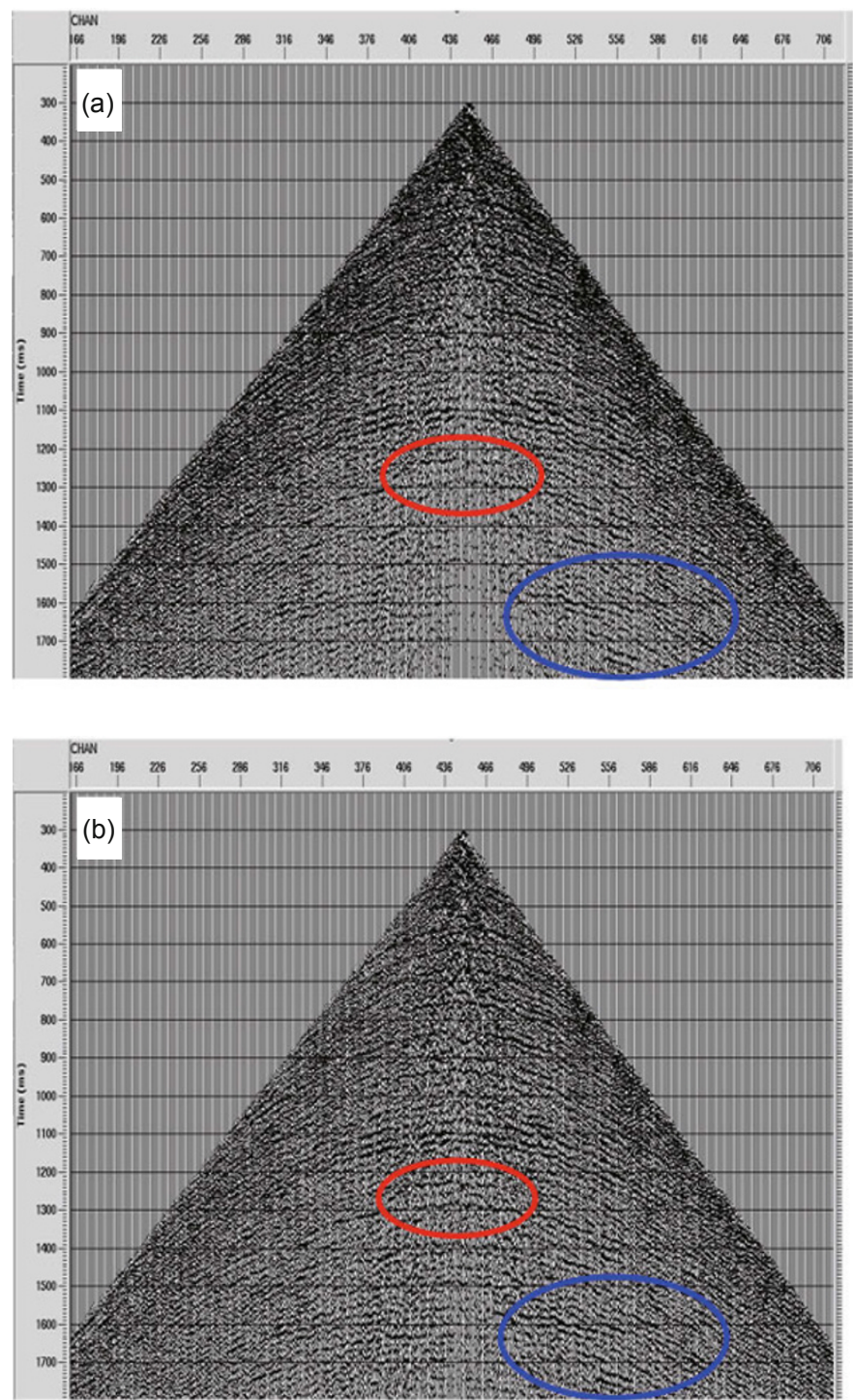

Fig. 14 Comparison of different processing results on Fig. 13 (a) Seismic section after frequency spectrum broadening processing on Fig. 13; (b) Seismic section after both multi-scale decomposition and frequency spectrum broadening processing on Fig. 13

\section{Conclusions}

1) When selecting the standard trace, traces with abundant signal frequency are preferred. The average amplitude spectrum of multi-channel data should be considered as well, to reduce the influence of random noise which is caused by using the standard trace only, and to make the compensation range more reasonable.

2) When compensating in the multi-scale domain with the spectral expansion method, the maximum variance norm criterion is introduced for selecting the appropriate simulating 
frequency band and the compensation effect is better.

3) The frequency spectrum fluctuates up and down, so fitting curves of different intervals are different. A best-fit curve should be determined by the median method to avoid the influence of choosing different intervals and to avoid the influence caused by differences in fitting curves on continuous compensation work.

A method to compensate seismic energy with multi-scale morphology is introduced in this paper. It is a new attempt and the result reaches the expected goal and gets a better processing effect of field data. The final result has higher resolution and better continuity of seismic events compared with other methods.

\section{References}

Chen $\mathrm{H}$, Guo $\mathrm{K}$ and $\mathrm{Hu}$ Y. A study on application of mathematical morphology to seismic signal processing. Progress in Geophysics. 2009. 24(6): 1995-2002 (in Chinese)

Chen H, Hu Y and Li J. Application of mathematical morphology to seismic fracture detection. Natural Gas Industry. 2008. 28(3): 48-50 (in Chinese)

Goutsias J and Heijmnas H J A M. Multi-resolution signal decomposition schemes, Part 2: Linear and morphological wavelets. IEEE Transactions on Image Processing. 2000. 9(11): 1877-1896

Koskinen L, Astola J and Neuvo Y. Soft morphological filters. Society of Optical Engineering. 1991. 1568: 262-270

Li K P, Li Y D, Zhang X G, et al. Stratigraphic absorption compensation based on wavelet packet decomposition. Geophysical Prospecting for Petroleum. 2000. 43(4): $542-549$ (in Chinese)

Li Q. Research on Application of Multi-scale Morphology in Seismic Data Processing. Beijing: China University of Petroleum (Beijing). 2005 (in Chinese)

Li Q Z. Toward the Road of Precise Exploration. Beijing: Petroleum Industry Press. 1993. 89-93, 115-116 (in Chinese)

Li Z C and Wang Q Z. A review of research on mechanism of seismic attenuation and energy compensation. Progress in Geophysics. 2007. 22(4): 1147-1152 (in Chinese)

Liu X W, Nian J B, Liu H, et al. Absorption attenuation compensation method based on generalized S transform. Geophysical Prospecting for Petroleum. 2006. 5(1): 9-14 (in Chinese)

Liu Z W and Wang Y C. A joint high-resolution processing method and its application for thin inter-beds. Petroleum Science. 2013. 10: 195204

Luo Y P, Jiang T, Wang X, et al. A new filtering method for LiDAR data based on mathematic morphological approach. Bulletin of Surveying and Mapping. 2011. 3: 15-19 (in Chinese)

McCarley and Lon A. An autoregressive filter model for constant $Q$ attenuation. Geophysics. 1985. 50(5): 749-758

Mukhopadhyay S and Chanda B. An edge preserving noise smoothing technique using multiscale morphology. Signal Processing. 2002. 8(2): 527-544

Ren H R, Wang H Z and Zhang L B. Compensation for absorption and attenuation using wave equation continuation along ray paths. Geophysical Prospecting for Petroleum. 2007. 46(6): 557-561 (in Chinese)

Sun W J and Fu L Y. Compensation for transmission losses based on one way propagators in the mixed domain. Geophysics. 2012. 77(3): 6572

Wang H Y and Sun Z D. Velocity dispersion and attenuation of seismic wave propagation in rocks. Acta Petrolei Sinica. 2012. 33(2): 332342 (in Chinese)

Wang J F. Research on Removing Regular Noise in Seismic Data with Morphology. Beijing: China University of Petroleum (Beijing). 2008a (in Chinese)

Wang J. Improve the resolution of vertical seismic profile with a travel time-varying inverse $Q$ filtering. Proceedings of $9^{\text {th }}$ International Conference on Signal Processing (ICSP 2008). Beijing, Oct. 26-29, $2008 b$

Wang R Q, Li Q and Zhang M. Application of multi-scaled morphology in denoising seismic data. Applied Geophysics. 2008. 5(3): 197-203

Wang S D. Attenuation compensation method based on inversion. Applied Geophysics. 2011. 8(2): 150-157

Wiggins R. Entropy guided deconvolution. Geophysics. 1985. 50(12): 2720-2726

Yu S P. High Resolution Seismic Exploration. Beijing: Petroleum Industry Press. 1993: 162-165 (in Chinese)

Yuan S Y and Wang S X. Spectral sparse Bayesian learning reflectivity inversion. Geophysical Prospecting. 2013. 61(4): 735-746

Yuan S Y and Wang S X. Influence of inaccurate wavelet phase estimation on seismic inversion. Applied Geophysics. 2011. 8(1): 48-59

Zhang G L, Xiong X J and Rong J J. Stratum absorption and attenuation compensation based on improved generalized S-transform. Oil Geophysical Prospecting. 2010. 45(4): 512-515 (in Chinese)

Zhang L B and Wang H Z. A stable inverse $Q$ migration method. Geophysical Prospecting for Petroleum. 2010. 49(2): 115-120 (in Chinese)

Zhang M, Sun Y P, Wei J M, et al. The study in the application of multiscale mathematical morphology in amplitude compensation of seismic data. Lecture Notes in Electrical Engineering. 2012. 126: 7-14

Zheng G J and Wang R Q. Exploration of application of multi-scale morphology in seismic data processing. Progress in Exploration Geophysics. 2003. 26(4): 277-281 (in Chinese)

Zhou H, Lin H and Sheng S B. High angle prestack depth migration with absorption compensation. Applied Geophysics. 2012. 9(3): 293-300

Zi L B and Gai Z X. Seismic inverse $Q$ filtering using Malvar window based time-frequency analysis. Journal of Peking University. 2012. 48(3): 405-410 (in Chinese)

(Edited by Hao Jie) 